\title{
SOBERANÍA ALIMENTARIA ¿Palabra de moda o concepto novedoso?
}

\author{
Frank Brassel ${ }^{1}$
}

\section{Resumen}

La Soberanía Alimentaria es un concepto que, desde la Asamblea Nacional Constituyente, juega un papel importante en los debates alrededor de una nueva política agraria y comercial en Ecuador. Es como la nueva palabra mágica en muchos debates pero en algunas ocasiones, no se sabe si se quiere dar sólo una nueva etiqueta a una política vieja. En otras, parece que se confunde la "soberanía alimentaria" con la "seguridad alimentaria" tradicional. ¿No es más que una palabra de moda? El autor argumenta que el concepto de la soberanía alimentaria es novedoso en la era posneoliberal del país y ofrece elementos relevantes en la búsqueda de una nueva política -sostenible y justa, apoyando a realizar los derechos del buen vivir- para el agro y para el país. El artículo intenta recuperar los orígenes del concepto, sus bases teóricas como su aplicabilidad en Ecuador y los retos en este camino.

\section{La agricultura en la época de la globalización neoliberal}

El concepto de soberanía alimentaria nació en los debates del movimiento campesino internacional sobre los impactos de la globalización neoliberal en la agricultura. El sector campesino fue discriminado tradicionalmente, pero con la filosofía y práctica neoliberal las economías campesinas fueron marginadas aún más. La apertura indiscriminada de mercados del Sur desde los años ochenta por los programas de ajuste del Fondo Monetario Internacional (FMI), debido a la introducción del tema agrario en la Organización Mundial de Comercio (OMC) y, recientemente, por los acuerdos bilaterales o regionales del llamado "libre comercio", enfrentó las economías

1 Historiador alemán, miembro del Comité Ejecutivo Internacional de FIAN, organización para el derecho a la alimentación. Trabaja desde hace 4 años como cooperante del EED (Servicio de las Iglesias Evangélicas en Alemania para el Desarrollo) en el SIPAE (Sistema de Investigación de la Problemática Agraria en Ecuador) en la Universidad Central del Ecuador. 
nacionales y especialmente las economías campesinas del Sur a una competencia desigual con la agroindustria del Norte. La desigualdad no está sólo basada en el poder de esta agroindustria, sino además en los subsidios gigantescos que recibe la misma por los Estados industrializados, mientras que las agriculturas campesinas del Sur se quedaron casi sin ningún apoyo público.

Los resultados son obvios: por un lado, se ha acelerado de forma intensa el poder de las corporaciones multinacionales en el agro $^{2}$ y de sus socios nacionales; por el otro, se ha verificado un empobrecimiento masivo y continuo en las zonas rurales del mundo, lo cual ha contribuido a una migración hacia los centros urbanos o los países industrializados. Según datos de un grupo de trabajo especializado de la ONU, el $80 \%$ de las 850 millones de personas en situación de hambre en el año 2005, vivieron en las zonas rurales donde se producen los alimentos. Más de la mitad de los hambrientos son familias de pequeños campesinos; otro cuarto está constituido por trabajadores agrícolas y campesinos sin tierra, y un $8 \%$ corresponde a pescadores, pastores y colectores de las frutas del bosque ${ }^{3}$. Obviamente, Ecuador es victima del mismo "desarrollo": la migración del campo, la caída dramática de las economías campesinas, el hecho que la mayoría de los hambrientos y de los pobres vive en el campo, la dependencia de importaciones de alimentos son indicadores de una crisis profunda que requiere respuestas nuevas.

\section{1. "Un futuro sin hambre"}

\section{-Origen y bases del concepto de la Soberanía Alimentaria}

La pobreza y el hambre se concentran en las zonas rurales, a la par que este espacio se vuelve dominio de la agroindustria. Ante esa constatación, la red internacional "Vía Campesina" introdujo el concepto de soberanía alimentaria durante la Cumbre contra el Hambre de la FAO en el año 1996. Para entender el con-

2 Ver por ejemplo el informe “¿De quién es la naturaleza? El poder coporativo y la frontera final en la mercantilizción de la vida", etcétera group, noviembre 2008, disponible en: http://www.etcgroup.org/es/los_ problemas/concentracion_corporativa.html

3 http://www.srfood.org/images/stories/pdf/officialreports/or-2-ungareporta63278en.pdf, p. 17 
cepto vale la pena re-leer en detalle la declaración con el título: "Soberanía Alimentaria, un futuro sin hambre"4.

"Vía Campesina es un movimiento creciente de organizaciones de campesinos, pequeños y medianos productores, trabajadores agrícolas, mujeres rurales y pueblos indígenas de todas las regiones del mundo. Sabemos que la seguridad alimentaria no puede lograrse sin tomar totalmente en cuenta a quienes producen los alimentos. Cualquier discusión que ignore nuestra contribución, fracasará en la erradicación de la pobreza y el hambre en las áreas rurales y urbanas".

Este principio de la "participación de los pequeños productores" en el cual insiste Vía Campesina es clave para cualquier política del agro, de desarrollo o de derechos humanos. Sobre esta base la declaración nombra sus propuestas y reivindicaciones fundamentales en cinco pilares.

\section{a) El derecho humano a la alimenta- ción}

"La alimentación es un derecho humano básico, todos y cada uno deben tener acceso a alimentos sanos, nutritivos y culturalmente apropiados, en cantidad y calidad suficientes para llevar una vida sana completa y con dignidad humana. Cada nación debe declarar el derecho de acceder a los alimentos como un derecho constitucional y garantizar el desarrollo del sector primario para asegurar la realización completa de este derecho fundamental”.

Entre los diferentes derechos económicos, sociales y culturales, el derecho a la alimentación ha recibido mayor atención en los ámbitos de la ONU, de organizaciones de derechos humanos y de movimientos sociales. El derecho a la alimentación se encuentra por primera vez en la Declaración Universal de los Derechos Humanos del año $1948^{5}$ y, más detalladamente, en el Pacto de los Derechos Económicos y Sociales del año 1996, el cual fue firmado por más de 150 naciones, el Ecuador de entre los primeros ${ }^{6}$. El desarrollo conceptual y práctico ha hecho del derecho a la alimentación un instrumento clave en la lucha contra el hambre y para un desarrollo social y

\footnotetext{
Detalles en: http://viacampesina.org/main_sp/

Ver: http://www.ohchr.org/SP o: http://www.fao.org/Legal/rtf/intl/intl-e.htm

Ver: http://www.unhchr.ch/tbs/doc.nsf/Statusfrset?OpenFrameSet
} 
ambientalmente sostenible en relaciones nacionales e internacionales ${ }^{7}$. Vale destacar, que a nivel internacional existe gran claridad que la práctica de este derecho fundamental no se realiza en primer lugar con programas de ayuda alimentaria, sino con políticas que facilitan el acceso de los pobres y hambrientos a recursos productivos para poder alimentarse ellos mismos. Esto nos lleva automáticamente al segundo pilar de la soberanía alimentaria.

\section{b) Reforma Agraria}

"Demandamos una auténtica Reforma Agraria que garantice a los sin tierra y familias campesinas -especialmente a las mujeres- la tenencia y el control de la tierra que trabajan y la devolución de los territorios a los pueblos indígenas. El derecho a la tierra debe estar libre de discriminación basada en género, religión, raza, clase social o ideología; la tierra pertenece a quienes la trabajan. Las familias campesinas, especialmente las mujeres, deben tener acceso a la tierra productiva, crédito, tecnología, mercados y servicios de extensión. Los gobiernos deben establecer y apoyar sistemas descentralizados de crédito rural que prioricen la producción de alimentos para el consumo doméstico y asegurar la soberanía alimentaría. Se debe usar la capacidad de producción, en lugar de la tierra como seguridad para garantizar el crédito".

Se incluyen tres elementos básicos en esta parte de la propuesta, relevantes para el debate ecuatoriano:

- Sin acceso a suficiente tierra no se pueden superar la pobreza y el hambre en el campo $^{8}$.

- El principio de la no-discriminación.

- El concepto no de una reforma agraria simplista, sino de una reforma integral que parte de una distribución más equitativa de la tierra, pero debe incluir un cambio de las políticas agrarias a favor de los pequeños productores.

7 Más información teórica y práctica de una implementación del derecho a la alimentación se encuentran en los documentos de la organización internacional FIAN, con este derecho como mandato: www.fian.org

8 Ver detalles en: Sofía Monsalve, Acceso a la tierra y los recursos productivos. Hacia una interpretación sistemática de las Directrices Voluntarias de la FAO sobre el Derecho a la Alimentación, FIAN, Heidelberg 2006, www.fian.org 
c) Preservación de los recursos naturales

Para Vía Campesina, el concepto de la soberanía alimentaria no es sólo una propuesta para la actualidad sino también a largo plazo, por eso se propuso éste como tercer pilar:

"La soberanía alimentaria requiere del cuidado y uso sostenible de los recursos naturales, especialmente de la tierra, el agua y las semillas. Nosotros, los que trabajamos la tierra, debemos tener el derecho de practicar el manejo sostenible de los recursos naturales y preservar la diversidad biológica. Esto se puede hacer únicamente desde una base económica fuerte con seguridad en la tenencia de la tierra, suelos saludables y uso reducido de productos agroquímicos. La sostenibilidad a largo plazo exige un cambio de la dependencia en los químicos y en los monocultivos de exportación y modelos de producción intensivos industrializados. Se requieren sistemas diversificados, naturales y equilibrados.

Los recursos genéticos son el resultado de milenios de años de evolución y pertenecen a toda la humanidad. Ellos representan el trabajo cuidadoso y el conocimiento de muchas generaciones de pueblos rurales e indígenas. Patentar y comercializar recursos genéticos por parte de compañías privadas y transnacionales debe ser prohibido. El acuerdo del Derecho de Propiedad Intelectual de la Organización Mundial del Comercio es inaceptable. Las comunidades campesinas tienen el derecho de usar libremente y proteger los recursos genéticos diversos, incluyendo las semillas, las cuales han sido desarrolladas por estas mismas comunidades a través de la historia. Esta es la base para lograr la soberanía alimentaria”.

Esta propuesta está basada en las experiencias largas de las familias campesinas e indígenas. Sólo desde la "revolución verde" se amplía un modelo no-sostenible, parcialmente también en las agriculturas campesinas. Ese es el modelo de la agroindustria, que ha reducido los alimentos, la tierra y el agua a meras mercancías, sin funciones sociales, ambientales y culturales. Por eso Vía Campesina diseña otro modelo frente al tema.

\section{d) Reorganización del comercio de ali- mentos}

"Los alimentos son, en primer lugar, una fuente de nutrición y, únicamente, en segundo lugar un artículo comercial. Las políticas agrícolas nacionales deben darle prioridad a la producción para el consumo doméstico y la autosuficiencia alimentaria. Los alimentos de exportación no 
Frank Brassel

deben desplazar a la producción local ni desplomar los precios. Esto significa que, el dumping por productos subsidiados para la exportación de alimentos debe cesar. Los campesinos tienen el derecho a producir alimentos básicos esenciales para sus países y controlar el mercado de sus productos. Los precios de los alimentos en los mercados nacionales e internacionales deben ser regulados y deben reflejar el verdadero costo de producción de los mismos".

Estas frases suenan muy actuales en esta época de diversas crisis internacionales, especialmente la del alza de los precios de los alimentos, la cual es la del modelo de la globalización neoliberal, bajo control de las empresas multinacionales y de las instituciones financieras internacionales. El dumping describe el fenómeno de las exportaciones agrarias de los países industrializados, las cuales se pueden ofrecer en los mercados de los países del Sur por debajo de sus costos de producción -y en muchos casos por debajo de los costos de producción en el Sur-por altísimos subsidios estatales a la producción y a la exportación de estos productos (por ejemplo maíz, trigo, leche, pollo, carnes, etcétera). Esta política persistente del dumping y de los subsidios de los Estados Unidos, de la Unión Europea y de Japón es la razón principal porque no se ha logrado un consenso en la OMC. Los países industrializados, simplemente se niegan a cambiar su política y buscan "alternativas" en forma de tratados del libre comercio en todos los continentes, con los cuales han logrado una apertura mayor de las economías del Sur sin cambiar en nada su política proteccionista y expansionista, de la que se aprovechan las grandes empresas multinacionales de los agronegocios.

La declaración de Vía Campesina formuló ya en el año 1996, como su quinto pilar de la soberanía alimentaria, una propuesta interesante de control de agroindustria internacional:

e) Eliminar la globalización del hambre, regular las instituciones y empresas multinacionales

"La soberanía alimentaria es socavada por las instituciones multilaterales y por el capital financiero especulativo. El control creciente de las compañías multinacionales sobre las políticas agrícolas del mundo ha estado propiciado por las políticas económicas de las instituciones multilaterales como la Organización Mundial del Comercio, el Fondo Monetario Internacional y el Banco Mundial. Exigimos regulación y esta- 
blecimiento de impuestos al capital especulativo y que sea implementado un estricto control mediante un código de conducta para las transnacionales".

Esta reivindicación ha ganado aún más relevancia con la crisis de los precios durante los últimos dos años. Su realización parece tan lejos como antes y tan necesaria como nunca. Frente a la "Conferencia de alto nivel sobre la seguridad alimentaria" en junio del año 2008 en Roma, las organizaciones de la sociedad civil pidieron " $\mathrm{Q} Q u e$ no se repitan los errores de siempre!" por la confirmación de una nueva agrupación bajo la ONU, la cual incluyó el Banco Mundial, el FMI y la OMC, instituciones cuales han contribuido en gran parte a la crisis. Las organizaciones de la sociedad civil propusieron una nueva comisión internacional con la integración de instituciones de investigación, de las mismas organizaciones campesinas y de los consumidores y pidieron "un cambio local y global de paradigma hacia la soberanía alimentaria”.

Los cinco pilares presentados formaron el concepto inicial de la sobe- ranía alimentaria y son válidos hasta hoy. El concepto se ha discutido ampliamente, fue profundizado por la misma Vía Campesina y también en cooperación con otras organizaciones. Precisamente, como consecuencia de la cumbre de Roma 1996, se formó el "Comité Internacional de Planificación de las ONG y OSC para la soberanía alimentaria" (CIP), el cual actúa hasta hoy como facilitador del proceso de debate, de cabildeo y de acción frente a la FAO, a la OMC y otras organizaciones internacionales que tratan el tema de agricultura y de alimentación ${ }^{9}$. Una discusión muy amplia y profunda se dio en el Foro de Nyeleni en febrero de 2007 en el país africano de Malí, primer Estado que incluyó la soberanía alimentaria en su Constitución ${ }^{10}$.

Algunos temas entraron con más detalle en los debates sobre la soberanía alimentaria, los más importantes son:

- El rol de la mujer rural y de los jóvenes;

- La perspectiva de la agroecología y la amenaza por la biotecnología;

- La migración; 
- El nexo con los consumidores urbanos;

- El poder creciente de las empresas multinacionales en el agro; $y$,

- La importancia de los derechos humanos.

Estos nuevos temas, no remplazan sino profundizan los cinco pilares fundamentales, los cuales forman una base interesante de una visión alternativa sobre el agro. La soberanía alimentaria parte de una crítica fuerte al modelo agrario global dominante y constituye un concepto propositivo para superar la crisis del modelo agroindustrial al nivel global ${ }^{11}$.

\section{Inseguridad alimentaria}

La FAO propaga desde los años ochenta el concepto de la "Seguridad Alimentaria". La definición más frecuentemente utilizada es la adoptada en la Cumbre contra el Hambre de la FAO, del año 1996, usada hasta hoy por esa institución en su Programa
Especial para la Seguridad Alimentaria:

"Existe seguridad alimentaria cuando todas las personas tienen en todo momento acceso físico y económico a suficientes alimentos inocuos y nutritivos, para satisfacer sus necesidades alimenticias y sus preferencias en cuanto a los alimentos, a fin de llevar una vida activa y sana"12.

Obviamente, este concepto es menos amplio que el de soberanía alimentaria. No se refiere al derecho a la alimentación, falta un enfoque en los pequeños productores y los $\sin$ tierra, y su participación en programas alimentarios. Asuntos estructurales sobre la tenencia de la tierra, el impacto del régimen agrario mundial y, del "libre comercio" quedan excluidos, porque son debates políticos conflictivos, en los cuales los gobiernos de los países industrializados, que dominan las decisiones en la $\mathrm{FAO}$, no quieren ceder nada.

El concepto de la seguridad alimentaria fracasó. Las cifras de los

11 Explicaciones más profundas del concepto de la soberanía alimentaria proveen los estudios siguientes: Windfuhr, Michael / Jonsén, Jenny 2005: Food Sovereignty. Towards Democracy in Localised Food Systems, ITDG Publishing und FIAN International, Warwickshire 2005, y Haydeé Carrasco / Sergio Tajada: Soberanía alimentaria: La libertad de elegir para asegurar nuestra alimentación, Lima: Soluciones Prácticas - ITDG, 2008

12 www.fao.org/spfs-pesa/mision-spfs/es/ 
hambrientos en el mundo en la época de la globalización no ha decrecido, con la crisis del alza de los precios su cifra explotó de 850 a 926 millones en el año 2007, para llegar a 963 millones el año pasado ${ }^{13}$. Eso es aún más escandaloso, porque se producen suficientes alimentos a nivel global, pero falta un acceso más equitativo para el acceso a ellos. La crisis de precios de los últimos años mostró que la especulación incontrolada en las grandes bolsas de alimentos y la demanda y producción creciente de los agrocombustibles hacen del sistema tradicional de producción y comercialización un riesgo para los países pobres y condena a más seres humanos a ser víctimas de violaciones del derecho a la alimentación. Existe una inseguridad alimentaria dramática, a nivel global y nacional.

\section{Soberanía alimentaria en la nueva Constitución ecuato- riana y en la práctica política del régimen actual}

Es un resultado interesante de esta crisis dramática que un concep- to desarrollado "desde abajo", desde los mismos productores y sus organizaciones, haya podido entrar en los grandes debates internacionales y también en Ecuador, cuya nueva Constitución ha incorporado el concepto. Se menciona por primera vez en el Capítulo Segundo sobre los "Derechos del 'buen vivir", donde el artículo 13 hace el siguiente señalamiento:

"Las personas y colectividades tienen derecho al acceso seguro y permanente a alimentos sanos, suficientes y nutritivos; preferentemente producidos a nivel local y en correspondencia con sus diversas identidades y tradiciones culturales. El Estado ecuatoriano promoverá la soberanía alimentaria".

Es obvia la conexión de los derechos humanos con la política pública en materia de la producción alimenticia. Lamentablemente, este vínculo se pierde parcialmente en el intento de la concretización del concepto de la soberanía alimentaria en el artículo relevante 281 . La introducción determina todavía una obligación estatal, como es el carácter de los derechos, diciendo:

13 UN 2009: Global economic turmoil intensifies the food crisis. Immediate action is required to secure adequate long-term global food supply, Fact Sheet der UN High Level Task Force on the Global Food Security Crisis: http://www.un.org/issues/food/taskforce/pdf/Global Economic.pdf 
"Soberanía alimentaria constituye un objetivo estratégico y una obligación del Estado para garantizar que las personas, comunidades pueblos y nacionalidades alcancen la autosuficiencia de alimentos sanos y culturalmente apropiados de forma permanente".

Los lineamientos siguientes que deben explicar esta obligación en su gran mayoría no se definen como derechos sino como medidas políticas. Veamos algunos ejemplos:

1. Impulsar la producción, transformación agroalimentaria y pesquera de las pequeñas y medianas unidades de producción, comunitarias y de la economía social y solidaria.

2. Adoptar políticas fiscales, tributarias y arancelarias que protejan al sector agroalimentario y pesquero nacional, para evitar la dependencia de importaciones de alimentos.

3. Promover políticas redistributivas que permitan el acceso del campesinado a la tierra, al agua y otros recursos productivos.

Lamentablemente, estas descripciones no contienen, por ejemplo, un derecho de los campesinos a la tierra y al agua, sino dicen sólo que se promoverán políticas respectivas, una formulación que no implica obligación ninguna. El Estado impulsará el trabajo de pequeñas $y$ medianas
UPAs, pero no garantiza legalmente su existencia frente a la expansión de las plantaciones de la agroindustria. La protección que se menciona en el punto 2 se refiere al sector agroalimentario entero, no a los pequeños productores.

Los otros aspectos mencionados en el artículo 281 también se leen como un catálogo de medidas posibles e interesantes para el agroecuatoriano -tecnología e investigación adecuada, financiamiento para la pequeña producción, agrobiodiversidad y saberes ancestrales, redes entre productores y consumidores, impedir prácticas monopólicos, evitar alimentos contaminados, etcétera-. Pero -repito-, no son formulaciones que definen obligaciones estatales sobre derechos de los ciudadanos campesinos.

Vale la pena mencionar que de la misma forma se presenta el artículo 282 sobre el uso y acceso a la tierra:

"El Estado normará el uso y acceso a la tierra que deberá cumplir la función social y ambiental. Un fondo nacional de tierras, establecido por ley, regulará el acceso equitativo de campesinos y campesinas a la tierra.

Se prohíbe el latifundio y la concentración de la tierra, así como el acaparamiento o privatización del agua y sus fuentes. 
El Estado regulará el uso y manejo del agua de riego para la producción de alimentos, bajo los principios de equidad, eficiencia y sostenibilidad ambiental".

Muy relevante, para cualquier politica de cambio y de la soberanía alimentaria, es el principio de la participación, el cual acepta la nueva Constitución en los artículos 61-65.

Los grandes avances de la nueva Constitución en materia de soberanía alimentaria son obvios, pero no se pueden ignorar sus limitaciones. Esta ambigüedad se refleja en la política legal y real del gobierno de Rafael Correa.

Ya antes de terminar la nueva Constitución, la Asamblea Nacional Constituyente aprobó, por pedido del Presidente, el "Mandato Agrario", con la intención de frenar los efectos de la crisis del alza de los precios. El Mandato se justificó con el lema de la soberanía alimentaria, pero en primer lugar fue un apoyo a los agronegocios, con la suspensión de impuestos para la importación de agroquímicos y de las contribuciones al SRI. La protesta contra el Mandato Agrario resultó en la coalición más amplia desde años entre los movimientos campesinos e indígenas, quienes presentaron un "Mandato Agrario Alternativo", que ni siquiera se discutió en la Asamblea Nacional Constituyente.

Con la aprobación de la Constitución, la Comisión Legislativa y de Fiscalización tenía la tarea de diseñar una Ley de Soberanía Alimentaria en un periodo de sólo cuatro meses. El debate sobre el tema mostró la falta de claridad y unidad en el propio gobierno y los grupos que le apoyan, además el proceso careció de una verdadera participación de la ciudadanía. Primero, se había contratado un grupo de técnicos para elaborar una propuesta, bajo coordinación de Manuel Chiriboga, ex jefe negociador para el TLC con los Estados Unidos. La propuesta encontró poco apoyo entre las organizaciones rurales, tampoco en el mismo gobierno. Por varios meses circularon diferentes propuestas de lado de organizaciones campesinas e indígenas, de ONG, de la Comisión Legislativa misma, etcétera. En el último momento salió la "Ley Orgánica del Régimen de Soberanía Alimentaria" (Publicada en el Registro Oficial No. 583, 5 de mayo de 2009), que básicamente garantiza los avances de la Constitución, pero deja la concretización de una política de soberanía alimentaria en manos del gobierno, de la futura Asamblea Nacional y de 
una nueva "Conferencia de Soberanía Alimentaria" de la sociedad civil con la tarea de proponer durante los próximos dos años una serie de leyes específicas, entre otros, sobre las temas siguientes:

1. Uso y acceso a las tierras, territorios, comunas;

2. Agrobiodiversidad y semillas;

3. Desarrollo agrario;

4. Agroindustria y empleo agrícola;

5. Sanidad animal y vegetal;

6. Acceso al crédito público;

7. Regulación del seguro y subsidio alimentario.

Lamentablemente, la Ley Orgánica fue debilitada por un veto del Presidente en importantes áreas como los agrocombustibles, restricciones para transgénicos y camaroneras y en materia de la participación de las organizaciones campesinas. Pero, no cabe duda, que el debate, diseño y la decisión sobre las respectivas leyes secundarias constituyen un chance y un reto para todas las organizaciones y personas interesadas en la soberanía alimentaria.

\section{Retos para la implementa- ción de la Soberanía Alimen- taria en Ecuador}

El mayor reto para la implementación de la soberanía alimentaria en Ecuador es el acceso desigual a los recursos productivos, especialmente, tierra y agua. Tomando los datos del último tercer. Censo Agropecuario sabemos que existen 535.309 fincas menores a 5 hectáreas, las cuales constituyen un $63,5 \%$ de todas las Unidades Productivas Agropecuarias (UPAs), pero controlan apenas el $6,3 \%$ de la superficie agrícola del Ecuador, exactamente 774.225 hectáreas. El promedio de las pequeñas UPAs estaba en 1,45 hectáreas en el año 2000. Estas cifras no reflejan los procesos desde la dolarización, las que según recientes estudios, muestran una fragmentación fuerte de la pequeña propiedad ${ }^{14}$. Significa que la gran mayoría de las familias campesinas hoy en día ya no disponen de suficiente tierra para vivir de la agricultura, para reproducirse socialmente. Hay que tomar en cuenta también una cifra desconocida de

14 Ver más detalles en: Brassel, Frank; Herrera, Stalin; Laforge, Michel (Editores): ¿Reforma Agraria en el Ecuador?: viejos temas, nuevos argumentos, SIPAE, Quito 2008 
campesinos sin tierra, quienes dependen completamente de trabajos precarios en las plantaciones o haciendas, en las ciudades o en la migración.

El desarrollo desde la apertura económica y del ajuste estructural en los años ochenta ha contribuido, por un lado, al auge de la agroindustria en Ecuador, y por otro a una "descampesinización"15 o "desestructuración excluyente"16 del campo. Durante la "larga noche neoliberal" el Estado dejó todos los programas para el fomento de la pequeña producción campesina, la cual perdió crecientemente sus bases de supervivencia, fue excluida en gran medida del desarrollo dominante. La pequeña producción fue aplastada por una agroindustria, la cual sí recibió apoyo de instancias nacionales e internacionales y, además, estaba bien conectada con los mercados globales. Durante este régimen del "mercado libre" se incrementó la injusticia en Ecuador, así lo prueba un equipo de investigadores bajo la coordinación de Carlos Larrea, quien analizando la década del año 1995 hasta 2006: concluye "Sorprendentemente, a pesar de este crecimiento económico, que alcanza el 7\% para todo el periodo, la pobreza declina solamente en 2 puntos porcentuales. El carácter concentrador del modelo virtualmente elimina las ventajas del reducido crecimiento alcanzado"17. Las cifras preparadas por el equipo de Larrea muestran que la pobreza persiste en el campo. "No se justifica por ningún motivo que un país de ingresos medios tenga una situación de desnutrición infantil tan grave como Ecuador la tiene", opinó Helmut Rauch, director del Programa Mundial de Alimentos de la ONU en Ecuador, en enero del año 2009 (El Telégrafo, 22 de enero de 2009). El $26 \%$ de la población infantil hasta 5 años en el país sufre de hambre crónica, en las zonas rurales la cifra sube a $35,5 \%$, y entre los niños indígenas llega a $40 \%$.

Sin medidas legales y políticas para cumplir con las normas del

15 Ver: SIPAE: Hacia una agenda para las economias campesinas en el Ecuador, Quito 2007

16 Ver: Rubio, Blanco: Explotados y Excluidos. Los campesinos latinoamericanos en la fase agroexportadora neoliberal, Quito 2009

17 Carlos Larrea (Coordinador): Mapas de pobreza, consumo por habitante y desigualdad social en el Ecuador: 1995-2006. Metodología y resultados, Quito 2008, p. 10. 
derecho a la alimentación no se podrá hablar de soberanía alimentaria. El hambre y la pobreza no se podrán resolver sin cambios estructurales en el campo. En el marco de este concepto hay que afrontar cuatro retos complejos y fundamentales:

1. Aplicar el derecho a la alimentación.

2. Implementar reformas redistributivas.

3. Regular la agroindustria.

4. Establecer una nueva política pública para el agro.

\subsection{Aplicar un sistema del derecho a la alimentación}

Los derechos humanos formulan obligaciones de los Estados ${ }^{18}$, las que tienen tres diferentes niveles: respetar, defender y realizar los dere$\operatorname{chos}^{19}$, y ofrecen, -en el caso clave del derecho a la alimentación- una buena herramienta para las organizaciones rurales y para el Estado a fin de que éstas aseguren la implementación de una política de soberanía alimentaria.
La obligación de respetar los derechos. Esto significa que el Estado no debe destruir el acceso de los campesinos a los recursos tierra y agua, que son la base de su supervivencia. Se refiere por ejemplo a desplazamientos forzados, como los sucedidos en el pasado ha causa de varias instituciones públicas, un caso de ellos es el INDA. Esto causa violaciones del derecho a la alimentación de las familias campesinas, también el derecho a tener un lugar de vivienda.

La obligación de proteger los derechos contra terceros. El Estado debe adoptar medidas para que terceros como terratenientes o empresas no violen los derechos de los campesinos. Por ejemplo, si una empresa minera destruye la base de los recursos naturales para campesinos y pueblos indígenas, el Estado debe cumplir con su obligación de proteger el derecho a la alimentación.

La obligación es garantizar los derechos humanos. El Estado debe dirigir el máximo de sus recursos disponibles para la realización progresiva de los derechos humanos.

18 La nueva Constitución ecuatoriana del año 2008 accepta explícitamente en su artículo 10 "los instrumentos internacionales" en materia de derechos humanos.

19 Ver el Comentario General sobre el derecho a la alimentación del Comité de Derechos Económicos, Sociales y Culturales de la ONU: "The right to adequate food (Art.11) 12/05/99. E/C.12/1999/5. (General Comments), Twentieth session, Geneva, 26 April-14 May 1999, en: http://www.unhchr.ch/tbs/doc.nsf 
Eso puede significar que el gobierno debe identificar en detalle a los campesinos sin tierra, como un grupo que requiere atención especial $y$, tomar medidas adecuadas para ellos, como la entrega de tierras para la producción de alimentos o salarios mínimos que alcancen a satisfacer sus necesidades.

Existen unas "Directrices Voluntarias en apoyo de la realización progresiva del derecho a una alimentación adecuada en el contexto de la seguridad alimentaria nacional" de la FAO de noviembre 2004, las que concretizan el derecho a la alimentación de una manera muy práctica para los gobiernos y para la sociedad civil20. Es obvio que el derecho a la alimentación no significa en primer lugar organizar programas de alimentación para los pobres. Eso es sólo una obligación del Estado en situaciones de catástrofes o para grupos de personas que no están en capacidad de trabajar para obtener sus alimentos, como niños huérfanos, enfermos o ancianos. Pero la idea principal es el derecho de alimentarse, para lo cual el Estado debería apoyar facilitando el acceso a puestos de trabajo dignos o medios de producción en la agricultura.

El acceso a la tierra es la base para lograr el derecho a la alimentación para las familias rurales sin tierra y para los pequeños productores ${ }^{21}$. Por eso, es obligación del Estado facilitar un acceso democrático a la tierra y al agua, como a los otros servicios productivos, dando prioridad a los sectores rurales más pobres. Reformas y políticas redistributivas para lograr este acceso deben ser una tarea permanente del Estado. Esto no es sólo un interés de las victimas de la política neoliberal en el campo, sino también del gobierno, porque se sabe que son, principalmente, los pequeños productores quienes producen alimentos para los mercados locales y nacionales, no para la exportación ${ }^{22}$.

20 http://www.fao.org/docrep/meeting/009/y9825s/y9825s00.htm

21 Ver la argumentación detallada y basada en los documentos de la historia de los derechos humanos en: Sofía Monsalve: Acceso a la tierra y los recursos productivos. Hacia una interpretación sistemática de las Directrices Voluntarias de la FAO sobre el Derecho a la Alimentación, FIAN Internacional, Heidelberg 2006, www.fian.org.

22 Ver los datos relevantes de la contribución de la agricultura familiar a la producción de alimentos en Ecuador y en la región: SIPAE: Hacia una agenda para las economias campesinas en el Ecuador, Quito 2007, pp. 42-47, y FAO/BID: Políticas para la Agricultura Familiar en América Latina y el Caribe, Santiago de Chile 2007 
"'Creemos que no puede haber una mejora en la desigualdad y una erradicación de la pobreza extrema y del hambre, si no hay un proceso que facilite el acceso a los medios de producción, al agua y las riquezas naturales' -resumió el representante de la FAO para América Latina, José Graciano, los debates respectivos durante la última Conferencia Regional en Brasil, abril 2008” (El Comercio, 15 de abril 2008).

\subsection{Implementar reformas redistri- butivas}

En las ultimas dos décadas de la política neoliberal en Ecuador se propagó el mito que ya no había ni la necesidad ni la disponibilidad de tierras para una nueva reforma agraria.
Tomando en cuenta la falta de tierras para la gran mayoría de los campesinos y la inequidad tremenda de la distribución de la tierra, no cabe duda, que una nueva reforma agraria integral es urgente. ¿Pero hay tierras para su distribución?

Estadísticamente, en Ecuador se dispone de casi 15 hectáreas de tierra por familia campesina o indígena. $\mathrm{Si}$ un acuerdo nacional facilitaría que de las UPAs mayores a 200 hectáreas proporcionen sólo el $20 \%$ de sus predios para un proceso de una nueva reforma agraria integral, los dueños de las grandes propiedades no caerían en pobreza, pero para las UPAs pequeñas, menores a 5 hectáreas, esta redistribución de alrededor de unas 720.000 hectáreas significaría que se

Gráfico 1

Distribución inequitativa de la tierra en Ecuador

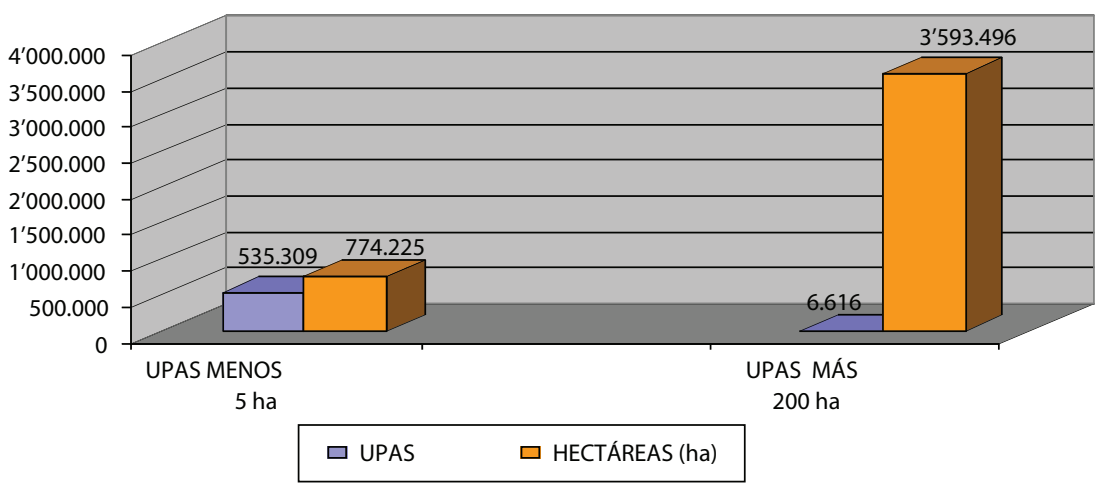

Fuente: III. Censo Agropecuario 
pueda duplicar su acceso a la tierra. Más concreto: la familia promedio que ahora apenas tiene 1,5 hectáreas podría acceder a unas 3 hectáreas, lo que sería una base para una producción viable. Mientras una pequeña minoría de 6.616 dueños dejaría apenas un $20 \%$ de sus extensiones grandes, y esto bajo ciertas modalidades de recompensación; una gran mayoría de más de 535 mil familias campesinas e indígenas ganaría un $100 \%$. Este primer cálculo ¿no podría ser la base de un debate nacional sobre una solución digna y duradera de la cuestión agraria en el Ecuador?

La nueva Constitución orienta de forma interesante hacia una política redistributiva: la función social y ambiental de la tierra. La tierra no es una mercancía sino la garantía para la soberanía y la supervivencia de un país. Por eso su distribución debe tener funciones sociales y ambientales. Debe servir en primer lugar para producir alimentos y crear puestos de trabajo. En los dos aspectos la agricultura familiar es más eficiente que la agroindustria. La nueva Ley Orgánica refuerza esta argumentación:

"Artículo 6. Acceso a la tierra.- El uso y acceso a la tierra deberá cumplir con la función social y ambiental. La función social de la tierra implica la generación de empleo, la redistribu- ción equitativa de ingresos, la utilización productiva y sustentable de la tierra. La función ambiental de la tierra implica que ésta procure la conservación de la biodiversidad y el mantenimiento de las funciones ecológicas; que permita la conservación y manejo integral de cuencas hidrográficas, áreas forestales, bosques, ecosistemas frágiles como humedales, páramos y manglares, que respete los derechos de la naturaleza y del "buen vivir"; y que contribuya al mantenimiento del entorno y del paisaje.

La ley que regule el régimen de propiedad de la tierra permitirá el acceso equitativo a ésta, privilegiando a los pequeños productores y a las mujeres productoras jefas de familia; constituirá el fondo nacional de tierras; definirá el latifundio, su extensión, el acaparamiento y concentración de tierras, establecerá los procedimientos para su eliminación y determinará los mecanismos para el cumplimiento de su función social y ambiental. Así mismo, establecerá los mecanismos para fomentar la asociatividad e integración de las pequeñas propiedades. Además, limitará la expansión de áreas urbanas en tierras de uso o vocación agropecuaria o forestal, así como el avance de la frontera agrícola en ecosistemas frágiles o en zonas de patrimonio natural, cultural y arqueológico, de conformidad con lo que establece el Art. 409 de la Constitución de la República”. 
Durante los próximos años se requiere un debate amplio, entre organizaciones campesinas, indígenas y pequeños productores, entre el gobierno y la sociedad civil sobre una distribución adecuada y factible de la tierra. ¿Se necesitan límites máximos de la propiedad de una persona -natural o jurídica-? ¿Cuál sería el tamaño óptimo de una UPA en las diferentes regiones del país? ¿Quiénes deberían tener prioridad en recibir nueva tierra? ¿Cómo fortalecer el rol de la mujer rural en la propiedad de la tierra? ¿Con cuáles métodos se puede evitar la fragmentación de las pequeñas UPAs? ¿Cómo financiar una nueva reforma agraria integral?

Para la elaboración de la nueva Ley de Tierras parece importante observar cuatro principios básicos:

1. El proceso debe ser mucho más participativo que en el caso de la Ley de Soberanía Alimentaria, sobre todo se requiere una participación activa y decisiva de las organizaciones campesinas e indígenas. Un tema con tanta importancia para toda la nación, también con tanta conflictividad requiere un consenso amplio.

2. La prioridad debe ser el derecho a la alimentación y, por eso, en consecuencia se debe garantizar el acceso a la tierra para los más pobres del campo. Un proceso que hace de los sin tierra meros beneficiarios de programas de alimentación, contradice la dignidad humana y no cumple con las normas de la soberanía alimentaría.

3. La función social de la tierra impide que se promueva un proceso de cambios en la estructura agraria en base a las lógicas del mercado, según los conceptos del Banco Mundial, pues experiencias de "reforma agraria de mercado" han fracasado en varios países ${ }^{23}$.

23 Las experiencias e investigaciones sobre la llamada "reforma agraria de mercado" ha evaluado en mayor detalle Saturnino M. Borras, Questioning Market-Led Agrarian Reform: Experiences from Brazil, Colombia and Sotuh Africa, en: Journal of Agrarian Change, Vol. 3, No.3, July 2003:367-394. Existe un resumen sobre el tema en castellano en Alerta Agraria No. 13 del SIPAE, Diciembre 2008, "La Reforma Agraria dirigida por el Mercado: alcances y límites de la propuesta del Banco Mundial en América Latina" (http://www.sipae.com/alerta_agraria.html) 
4. Pero además, hace falta establecer claras regulaciones a los mercados de tierra, para evitar que sigan desarrollando procesos de re-concentración o de fragmentación de la propiedad agraria.

Vale la pena mencionar que una re-distribución de la tierra, como la propone también la nueva Constitución del Ecuador, sería una contribución a la democratización de la sociedad y un paso importante para superar la "larga noche neoliberal" y el legado colonial.

\subsection{Regular la agroindustria}

Como se mencionó anteriormente, la agroindustria era el aspecto ganador de la época neoliberal en Ecuador-como en casi todos los países del mundo-. Este proceso se desarrolló a costo de la agricultura familiar y causó graves violaciones de los derechos de familias campesinas e indígenas a tierra y agua ${ }^{24}$, como de los derechos laborales y ambientales. Por eso es clave una regulación de la agroindustria; es un logro importante de las pocas organizaciones que trabajan el tema que la Ley Orgánica defina unas pautas importantes para tratar el tema en la futura Ley de Agroindustria:

"Artículo 17. Leyes de Fomento a la Producción.- ...El Estado garantizará una planificación detallada y participativa de la política agraria y del ordenamiento territorial de acuerdo al Plan Nacional de Desarrollo, preservando las economías campesinas, estableciendo normas claras y justas respecto a la operación y del control de la agroindustria y de sus plantaciones para garantizar equilibrios frente a las economías campesinas, y respeto de los derechos laborales y la preservación de los ecosistemas".

Se sabe que en el pasado, hasta en la actualidad, la agroindustria actuaba básicamente "fuera de la ley". Obviamente, será un reto grande cambiar esta tradición, pero sin este cambio habrá sólo poco espacio para el desarrollo de las economías campesinas las que son la base de la política de la soberanía alimentaria. Ecuador?: viejos temas, nuevos argumentos, SIPAE, Quito 2008. 
4.4 Nueva política pública para el agro, en el marco de una estrategia de soberanía alimentaria

Diseñar un sistema de soberanía alimentaria es un reto mayor en la segunda fase de la "revolución ciudadana" del presidente Rafael Correa. Los tres pilares ya mencionados son la base de una política que toma la soberanía alimentaria no sólo como un nuevo lema, sino como un concepto novedoso para el agro y el desarrollo de Ecuador. Eso debe ser un proceso ampliamente participativo, se requiere un debate, el diseño y la implementación de una nueva politica agraria. Los artículos 281 y 282 de la nueva Constitución ofrecen una importante orientación.

Es obvio, que un verdadero cambio sólo puede darse a partir de un Estado activo que fomente y proteja la pequeña y mediana producción. Actualmente, SENPLADES busca integrar el concepto de la soberanía alimentaria en el nuevo Plan de
Desarrollo hasta el 2025. En las políticas prácticas se tratará seguramente de dinamizar el Ministerio de Agricultura, el cual casi no ha cambiado sus orientaciones en los últimos años.

La sostenibilidad de la agricultura debe jugar un papel importante para el futuro. La agroecología y los saberes tradicionales deben ganar mucho más peso, un aspecto en el cual el país tiene ventajas comparativas a nivel internacional. El fomento de la pequeña producción, apoyado por compras públicas, investigación, créditos y asistencia técnica, debe ser una base de la nueva política agraria, así mismo como la concientización de los consumidores, alianzas entre consumidores y productores, el control de importaciones agropecuarias en materia de salud y de interés nacional. El interés de la política comercial debe ser apoyar en la realización de los derechos del buen vivir, donde la soberanía alimentaria es parte importante. 